\title{
Self-Management Improves Asthma
}

Janson SL, McGrath KW, Covington JK, et al.: Individualized asthma self-management improves medication adherence and markers of asthma control. J Allergy Clin Immunol 2009, 123:840-846.

Rating: • Of importance.

Introduction: Adherence to asthma medications is essential for achieving asthma control and reducing exacerbations. This study examined whether self-management education improved medication adherence.

Aims: The aim of this study was to determine whether selfmanagement education affects long-term adherence to inhaled corticosteroid therapy and markers of asthma control.

Methods: The study was a single-center, randomized controlled trial of 95 adults (ages 18-55 years) with moderate to severe persistent asthma conducted over 22 weeks. The patients were randomly assigned to receive individualized self-management education or usual asthma care. The three self-management intervention sessions occurred during 30 minutes and were provided by an advanced practice nurse or respiratory therapist who was a certified asthma educator. The sessions included general asthma education, assessment and correction of inhaler technique, initiation of an individualized action plan, and individualized environmental control strategies.

Results: Patients who were randomly assigned to the selfmanagement group had greater inhaled corticosteroid adherence, improved perceived control of asthma, fewer nighttime awakenings, and a decreased need for inhaled $\beta$-agonists compared with those in the control group.

Discussion: This trial showed that individualized asthma self-management improves clinical markers of asthma and medication adherence.

\section{Comments}

Although this study examined an adult population, it provides further evidence that self-management skills should be considered by all clinics for their patients with asthma.

\section{Acknowledgment}

Drs. Christina E. Ciaccio and Jay M. Portnoy are affiliated with the Section of Allergy, Asthma, and Immunology at the Children's Mercy Hospitals and Clinics, where Dr. Ciaccio is an Assistant Professor of Pediatrics and Dr. Portnoy is the Chief of the Section of Allergy, Asthma, and Immunology and a Professor of Pediatrics. Correspondence should be sent to 2401 Gillham Road, Kansas City, MO 64108, USA. E-mail: ceciaccio@cmh.edu; jportnoy@cmh.edu.

\section{Disclosure}

No potential conflicts of interest relevant to this article were reported. 